\title{
Ácidos fenólicos como antioxidantes
}

\section{Phenolic acids as antioxidants}

Sergio Eduardo SOARES'

RES U M O

Os compostos fenólicos têm sido muito estudados devido a sua influência na qualidade dos alimentos. Englobam uma gama enorme de substâncias, entre elas os ácidos fenólicos, os quais, por sua constituição química, possuem propriedades antioxidantes. Assim, a presente revisão procura reunir diversos estudos que avaliaram o potencial antioxidante dos ácidos fenólicos na conservação de alimentos lipídicos. Além disso, são reunidos também estudos sobre a ação antioxidante destes compostos no sistema biológico através da neutralização dos radicais livres gerados no organismo, que estão associados a diversas doenças como câncer e doenças cardiovasculares.

Termos de indexação: ácidos fenólicos, oxidação lipídica, radicais livres, antioxidantes, conservação de alimentos.

\section{A B S T R A C T}

The phenolic compounds have been widely studied due to their influence on food quality. They are constituted by a large amount of substances, among them the phenolic acids, which have antioxidant properties, as a result of their chemical structure. So, the present review collects several papers that evaluated the antioxidant potential of the phenolic acids for the lipid foods preservation. This study also collects papers about the antioxidant action of these compounds in biological systems through the neutralization of free radicals produced in the organism, which are associated with many pathologies like cancer and heart diseases.

Index terms: phenolic acids, lipid oxidation, free radicals, antioxidants, food preservation.

\footnotetext{
1 Faculdade de Ciências da Saúde, Universidade de Marília. Av. Hygino Muzzi Filho, 1001, 17525-902, Marília, SP, Brasil. E-mail:ssoares@mii.zaz.com.br
} 


\section{N T R O D U Ç Ã O}

A presença dos compostos fenólicos em plantas tem sido muito estudada por estes apresentarem atividades farmacológica e antinutricional e também por inibirem a oxidação lipídica e a proliferação de fungos (Nagem et al., 1992; Gamache et al., 1993; Ivanova et al., 1997; Aziz et al., 1998; Fernandez et al., 1998; Hollman \& Katan, 1998), além de participarem de processos responsáveis pela cor, adstringência e aroma em vários alimentos (Peleg et al., 1998).

Diversos pesquisadores têm trabalhado na separação, identificação, quantificação e utilização dos compostos fenólicos em alimentos, enfrentando muitos problemas metodológicos, pois, além de englobarem em uma gama enorme de substâncias (fenóis simples, ácidos fenólicos, cumarinas, flavonóides, taninos e ligninas), eles são, na maioria das vezes, de grande polaridade, muito reativos, e suscetíveis à ação de enzimas (King \& Young, 1999).

Os ácidos fenólicos são algumas das substâncias que constituem o grupo dos compostos fenólicos. Caracterizam-se por terem um anel benzênico, um grupamento carboxílico e um ou mais grupamentos de hidroxila e/ou metoxila na molécula, conferindo propriedades antioxidantes tanto para os alimentos como para o organismo, sendo, por isso, indicados para o tratamento e prevenção do câncer, doenças cardiovasculares e outras doenças (Kerry \& Abbey, 1997; Bravo, 1998; Croft, 1998; Ferguson \& Harris, 1999).

Assim, esta revisão traz uma breve descrição da química dos ácidos fenólicos e de sua utilização como antioxidantes em alimentos e em sistemas biológicos.

\section{COMPOSTOS FENÓLICOS}

Os compostos fenólicos englobam desde moléculas simples até outras com alto grau de polimerização (Bravo, 1998). Estão presentes nos vegetais na forma livre ou ligados a açúcares (glicosídios) e proteínas (Croft, 1998).

Ribéreau-Gayon (1968) adotou a seguinte classificação para estes compostos: pouco distribuídos na natureza, polímeros e largamente distribuídos na natureza.

Na família dos compostos fenólicos pouco distribuídos na natureza estão um número reduzido deles, embora estes sejam encontrados com certa freqüência. Neste grupo estão os fenóis simples, o pirocatecol, a hidroquinona e o resorcinol. A esta família também pertencem os aldeídos derivados dos ácidos benzóicos, que são constituintes dos óleos essenciais como a vanilina.

Alguns compostos fenólicos não se apresentam em forma livre nos tecidos vegetais. São aqueles presentes sob a forma de polímeros, na qual estão os taninos e as ligninas. Os taninos são compostos de alto peso molecular, que conferem ao alimento a sensação de adstringência, e classificam-se em dois grupos, baseados em seu tipo estrutural: taninos hidrolisáveis e taninos condensados. Os primeiros contêm um núcleo central de glicose ou um álcool poliídrico, esterificado com ácido gálico ou elágico, e são prontamente hidrolisáveis com ácidos, bases ou enzimas. Os outros são polímeros de catequina e/ou leucoantocianidina, não prontamente hidrolisáveis por tratamento ácido. As ligninas são polímeros complexos de grande rigidez e resistência mecânica, e sua hidrólise alcalina libera uma grande variedade de derivados dos ácidos benzóico e cinâmico.

Na família dos compostos largamente distribuídos na natureza estão os fenólicos encontrados geralmente em todo o reino vegetal, mas às vezes podem estar localizados em uma só planta. Estes fenólicos estão divididos em dois grandes grupos: os flavonóides e derivados e os ácidos fenólicos (ácidos benzóico, cinâmico e seus derivados) e cumarinas.

Os flavonóides possuem uma estrutura básica formada por $\mathrm{C}_{6}-\mathrm{C}_{3}-\mathrm{C}_{6}$, sendo os compostos mais diversificados do reino vegetal. Neste grupo encontram-se as antocianidinas, flavonas, 
flavonóis e, com menor freqüência, as auronas, calconas e isoflavonas, dependendo do lugar, número e combinação dos grupamentos participantes da molécula.

Os ácidos fenólicos são divididos em três grupos. O primeiro é composto pelos ácidos benzóicos, que possuem sete átomos de carbono $\left(C_{6}-C_{1}\right)$ e são os ácidos fenólicos mais simples encontrados na natureza; suas fórmulas gerais e denominações estão representadas na Figura 1. O segundo é formado pelos ácidos cinâmicos, que possuem nove átomos de carbono $\left(\mathrm{C}_{6}-\mathrm{C}_{3}\right)$, sendo sete os mais comumente encontrados no reino vegetal (Figura 2).

As cumarinas são derivadas do ácido cinâmico por ciclização da cadeia lateral do ácido o-cumárico (Figura 3).

Os ácidos fenólicos, além de se apresentarem sob sua forma natural, podem também se ligar entre si ou com outros compostos. A combinação mais importante destes ácidos ocorre com o ácido caféico, o qual, associado a um álcool-ácido cíclico, denominado ácido quínico, origina o ácido clorogênico.

\section{Oxidação lipídica nos alimentos}

Os lipídios nos alimentos estão sujeitos a uma série de reações que podem levar a modificações de suas estruturas, afetando o valor nutricional e também os padrões de qualidade, como cor, odor, sabor e textura (Hsieh \& Kinsella, 1989; Donnelly \& Robinson, 1995).

A oxidação lipídica é uma das principais reações deteriorativas a ocorrerem durante $\mathrm{o}$ processamento, distribuição, armazenamento e preparo final dos alimentos. É responsável pelo desenvolvimento de sabores e odores desagradáveis nos alimentos, tornando-os impróprios para o consumo. Além disso, ela também provoca outras alterações que irão afetar a qualidade nutricional, a integridade e a segurança dos alimentos, através da formação de compostos potencialmente tóxicos (Frankel, 1980; Nawar, 1985; Aruoma, 1993; Kubow, 1993).

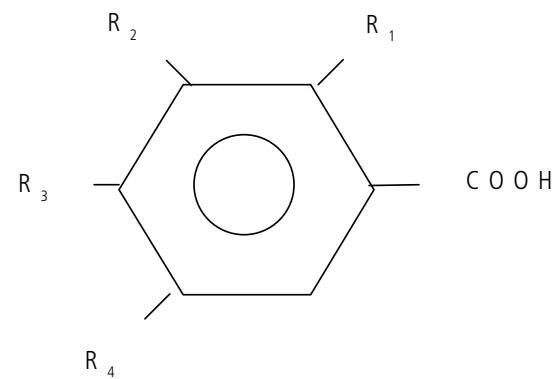

$\mathrm{R}_{1}=\mathrm{OH} \rightarrow$ Ácido Salicílico; $\mathrm{R}_{1}=\mathrm{R}_{4}=\mathrm{OH} \rightarrow$ Ácido Gentísico; $\mathrm{R}_{3}=\mathrm{OH} \rightarrow$ Ácido p-hidroxibenzóico; $\mathrm{R}_{2}=\mathrm{R}_{3}=\mathrm{OH} \rightarrow$ Ácido Protocatequínico; $\mathrm{R}_{2}=\mathrm{OCH}_{3 i}$ $\mathrm{R}_{3}=\mathrm{OH} \rightarrow$ Ácido Vanílico; $\mathrm{R}_{2}=\mathrm{R}_{3}=\mathrm{R}_{4}=\mathrm{OH} \rightarrow$ Ácido Gálico; $\mathrm{R}_{2}=\mathrm{R}_{4}=\mathrm{OCH}_{3^{\prime}}$; $\mathrm{R}_{3}=\mathrm{OH} \rightarrow$ Ácido Siríngico

Figura 1. Estrutura química dos ácidos benzóicos.

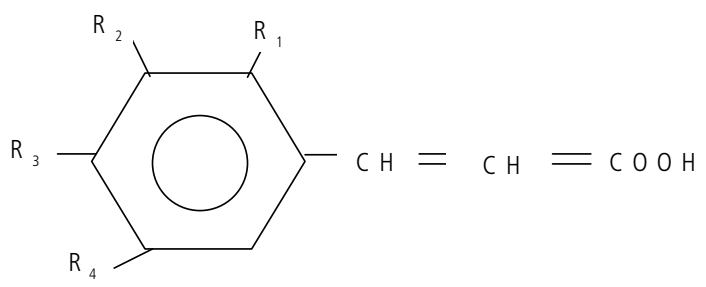

$R_{1}=R_{2}=R_{3}=R_{4}=H \rightarrow$ Ácido cinâmico; $R_{1}=\mathrm{OH} \rightarrow$ Ácido o-cumárico; $\mathrm{R}_{2}=\mathrm{OH} \rightarrow$ Ácido m-cumárico; $\mathrm{R}_{3}=\mathrm{OH} \rightarrow$ Ácido $\mathrm{p}$-cumárico; $\mathrm{R}_{2}=\mathrm{R}_{3}=$ $\mathrm{OH} \rightarrow$ Ácido Caféico; $\mathrm{R}_{2}=\mathrm{OCH}_{3} ; \mathrm{R}_{3}=\mathrm{OH} \rightarrow$ Ácido Ferúlico; $\mathrm{R}_{2}=\mathrm{R}_{4}=$ $\mathrm{OCH}_{3} ; \mathrm{R}_{3}=\mathrm{OH} \rightarrow$ Ácido Sinápico

Figura 2. Estrutura química dos principais ácidos cinâmicos.
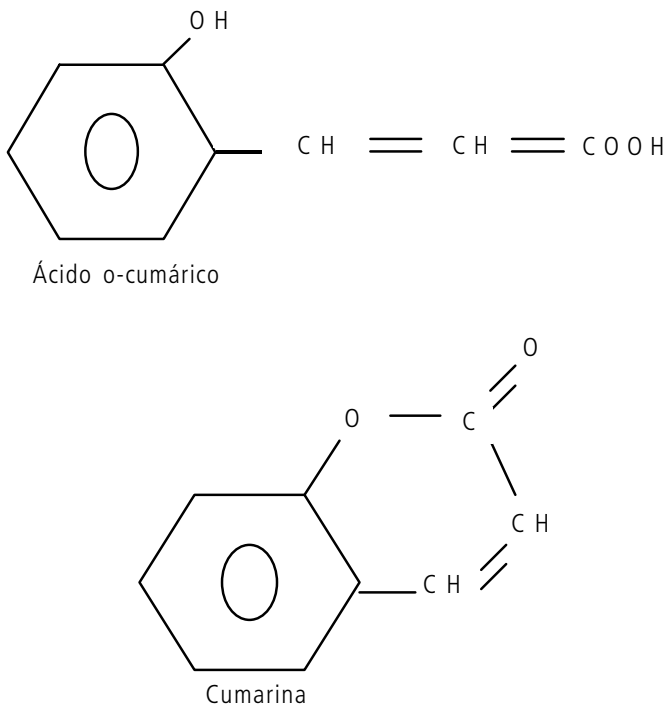

Figura 3. Estrutura química das cumarinas. 
Os ácidos graxos insaturados são as estruturas mais suscetíveis ao processo oxidativo, havendo uma dependência direta entre o grau de insaturação e a susceptibilidade à oxidação (Cosgrove et al., 1987).

\section{Oxidação em sistemas biológicos}

A oxidação nos sistemas biológicos ocorre devido à ação dos radicais livres no organismo. Estas moléculas têm um elétron isolado, livre para se ligar a qualquer outro elétron, e por isso são extremamente reativas. Elas podem ser geradas por fontes endógenas ou exógenas. Por fontes endógenas, originam-se de processos biológicos que normalmente ocorrem no organismo, tais como: redução de flavinas e tióis; resultado da atividade de oxidases, cicloxigenases, lipoxigenases, desidrogenases e peroxidases; presença de metais de transição no interior da célula e de sistemas de transporte de elétrons. Esta geração de radicais livres envolve várias organelas celulares, como mitocôndrias, lisossomos, peroxissomos, núcleo, retículo endoplasmático e membranas (Machlin \& Bendich, 1987). As fontes exógenas geradoras de radicais livres incluem tabaco, poluição do ar, solventes orgânicos, anestésicos, pesticidas e radiações.

Nos processos biológicos há formação de uma variedade de radicais livres (Erenel et al., 1993; Rice-Evans \& Burdon, 1993). São eles:

- Radicais do oxigênio ou espécies reativas do oxigênio

Íon superóxido $\left(\mathrm{O}_{2}^{-\bullet}\right)$

Hidroxila $\left(\mathrm{OH}^{\bullet}\right)$

Peróxido de hidrogênio $\left(\mathrm{H}_{2} \mathrm{O}_{2}\right)$

Alcoxila $\left(\mathrm{RO} \mathrm{O}^{*}\right)$

Peroxila (ROO*)

Peridroxila $\left(\mathrm{HOO}^{*}\right)$
Oxigênio sinlete $\left({ }^{1} \mathrm{O}_{2}\right)$

- Complexos de Metais de Transição

$\mathrm{Fe}^{+3} / \mathrm{Fe}^{+2}$

$\mathrm{Cu}^{+2} / \mathrm{Cu}^{+}$

- Radicais de Carbono

Triclorometil $\left(\mathrm{CCl}_{3}{ }^{\circ}\right)$

Radicais de Enxofre

Tiol (RS*)

- Radicais de Nitrogênio

Fenildiazina $\left(\mathrm{C}_{6} \mathrm{H}_{5} \mathrm{~N}=\mathrm{N}^{\bullet}\right)$

Óxido nítrico (NO*)

Estes radicais irão causar alterações nas células, agindo diretamente sobre alguns componentes celulares. Os ácidos graxos poliinsaturados das membranas, por exemplo, são muito vulneráveis ao ataque de radicais livres. Estas moléculas desencadeiam reações de oxidação nos ácidos graxos da membrana lipoprotéica, denominadas de peroxidação lipídica, que afetarão a integridade estrutural e funcional da membrana celular, alterando sua fluidez e permeabilidade. Além disso, os produtos da oxidação dos lipídios da membrana podem causar alterações em certas funções celulares (Rice-Evans \& Burdon, 1993). Os radicais livres podem provocar também modificações nas proteínas celulares, resultando em sua fragmentação, cross linking, agregação e, em certos casos, ativação ou inativação de certas enzimas devido à reação dos radicais livres com aminoácidos constituintes da cadeia polipeptídica. A reação de radicais livres com ácidos nucléicos também foi observada, gerando mudanças em moléculas de DNA e acarretando certas aberrações cromossômicas (Erenel et al., 1993). Além destes efeitos indiretos, há a ação tóxica resultante de altas concentrações de íon superóxido e peróxido de hidrogênio na célula (Halliwell et al., 1995). 


\section{COMPOSTOS FENÓLICOS COMOANTIOXIDANTES}

Os processos oxidativos podem ser evitados através da modificação das condições ambientais ou pela utilização de substâncias antioxidantes com a propriedade de impedir ou diminuir o desencadeamento das reações oxidativas (Allen \& Hamilton, 1983).

Os antioxidantes são capazes de inibir a oxidação de diversos substratos, de moléculas simples a polímeros e biossistemas complexos, por meio de dois mecanismos: o primeiro envolve a inibição da formação de radicais livres que possibilitam a etapa de iniciação; o segundo abrange a eliminação de radicais importantes na etapa de propagação, como alcoxila e peroxila, através da doação de átomos de hidrogênio a estas moléculas, interrompendo a reação em cadeia (Namiki, 1990; Simic \& Javanovic, 1994).

Antioxidantes fenólicos funcionam como seqüestradores de radicais e algumas vezes como quelantes de metais (Shahidi et al., 1992), agindo tanto na etapa de iniciação como na propagação do processo oxidativo. Os produtos intermediários, formados pela ação destes antioxidantes, são relativamente estáveis devido à ressonância do anel aromático apresentada por estas substâncias (Nawar, 1985). Os compostos fenólicos e alguns de seus derivados são, portanto, eficazes para prevenir a oxidação lipídica; entretanto, poucos são os permitidos para o uso em alimentos, devido principalmente a sua toxicidade (Shahidi et al., 1992).

$\mathrm{Na}$ indústria de alimentos, a oxidação lipídica é inibida por seqüestradores de radicais livres. Neste caso, os compostos mais utilizados, entre outros, são: butil-hidroxi-anisol (BHA), butil-hidroxi-tolueno $(\mathrm{BHT})$, tércio-butil-hidroxiquinona (TBHQ), tri-hidroxi-butilfenona (THBP) e propil galato (PG). Estudos toxicológicos têm demonstrado a possibilidade de estes antioxidantes apresentarem algum efeito tóxico, e o Joint Expert Committee on Food Aditives (JECFA ) da Food and Agriculture Organization
(FAO) e World Health Organization (WHO) têm alterado nos últimos anos a ingestão diária aceitável (IDA) destas substâncias como resultado de algumas pesquisas científicas (Würtzen, 1990).

Tendo em vista os indícios de problemas que podem ser provocados pelo consumo de antioxidantes sintéticos, as pesquisas têm-se dirigido no sentido de encontrar produtos naturais com atividade antioxidante os quais permitirão substituir os sintéticos ou fazer associações entre eles, com o intuito de diminuir sua quantidade nos alimentos. Os estudos estão centralizados nos compostos fenólicos de origem vegetal, pois eles agem como aceptores de radicais livres, interrompendo a reação em cadeia provocada por estes, além de atuarem também nos processos oxidativos catalizados por metais, tanto in vitro, como in vivo (Ho, 1992; Huang \& Ferraro, 1994; Nakatani, 1992; Pratt, 1992; Ho et al., 1994; Donnelly \& Robinson, 1995; Cintra \& Mancini Filho, 1996; Williamson et al., 1998).

\section{Ácidos fenólicos como antioxidantes em alimentos}

Diversos autores realizaram estudos visando verificar o potencial antioxidante dos ácidos fenólicos, com o objetivo de substituir os antioxidantes sintéticos, largamente utilizados na conservação de alimentos lipídicos por chegarem a aumentar a vida útil de muitos produtos entre 15 e 200\% (Durán \& Padilla, 1993).

Em investigações de ácidos fenólicos presentes em grãos de soja, farinha de soja desengordurada, concentrado e isolado protéico de soja (Pratt \& Birac, 1979) e dos ácidos cinâmicos encontrados nestes produtos, quatro apresentaram uma atividade antioxidante significativa. São eles: ácido clorogênico (encontrado em maior quantidade e com maior atividade antioxidante), caféico, $p$-cumárico e ferúlico. Este fato foi também observado em nove cultivares de soja produzidos no Brasil, entre os quais o UFV 5', com a maior concentração destes 
ácidos fenólicos; neste caso o ácido ferúlico apresentou a maior atividade antioxidante (Nagem et al., 1992).

Medindo a eficiência de alguns ácidos fenólicos como antioxidantes em sistema lipídico, através do Rancimat ${ }^{\circledR}$, verificou-se que os ácidos protocatequínico, caféico, ferúlico, gálico e sinápico possuem uma boa atividade (Dziedzic \& Hudson, 1984), embora estes compostos, assim como os demais ácidos fenólicos, possuam baixa solubilidade neste sistema, limitando em parte, sua utilização e seu potencial antioxidante (Pratt, 1992). Contudo, estas substâncias podem ser modificadas para se tornarem lipossolúveis através de alquilação ou esterificação com ácidos graxos de cadeia longa ou álcoois. Ademais, em avaliação do potencial dos ácidos caféico, protocatequínico, p-hidroxibenzóico, ferúlico e p-cumárico em sistema lipídico, alguns deles, como o caféico e o protocatequínico, apresentaram atividade antioxidante maior que o $\alpha$-tocoferol e o BHT (Von Gadow et al., 1997).

A atividade antioxidante da fração polar contida em óleo de oliva refinado foi testada nele mesmo através do método de Shall (estufa a $60^{\circ} \mathrm{C}$, na ausência de luz), determinando-se o índice de peróxidos como indicador do processo oxidativo (Papadopoulos \& Boskou, 1991). O extrato contendo a fração polar é constituído praticamente por compostos fenólicos, em particular por ácidos fenólicos. Os resultados mostraram uma certa ação por parte da fração polar do óleo de oliva, mas esta foi inferior a do BHT. Foi testada também a atividade antioxidante de cada ácido fenólico contido na fração polar, individualmente, utilizando a mesma metodologia. O ácido caféico mostrou uma atuação maior que a do BHT, enquanto os ácidos protocatequínico e siríngico, apesar de terem apresentado atividade, foram inferiores a do BHT. Por outro lado, os ácidos o-cumárico, p-cumárico, $p$-hidroxibenzóico e vanílico demonstraram pouca ou nenhuma propriedade antioxidante.

Em estudos da atividade antioxidante de extrato de farelo de trigo através do método do oxigênio ativo (Onyeneho \& Hettiarachchy, 1992), este mostrou um grande potencial. Após sua análise por Cromatografia Líquida de Alta Eficiência, detectaram-se quantidades apreciáveis de ácidos fenólicos, como protocatequínico, gentísico, caféico, vanílico, clorogênico e ferúlico.

Extratos de seis variedades de batata também foram submetidos ao método do oxigênio ativo, tendo o óleo de oliva como substrato (Onyeneho \& Hettiarachchy, 1993). Os resultados mostraram que em todos eles houve uma redução da oxidação, embora inferior à obtida com o BHA, BHT, TBHQ e com o extrato de alecrim. Estes extratos foram submetidos, ainda, à cromatografia em camada delgada para identificação dos compostos presentes e também foram revelados com $\beta$-caroteno/ácido linoléico para se testar a atividade antioxidante destes compostos. Os resultados indicaram serem os ácidos fenólicos os principais responsáveis pela atividade antioxidante destes extratos e serem os ácidos clorogênico, caféico e protocatequínico os mais ativos.

Em estudos realizados com extratos de casca de batata, através do método de Shall, foi observado que estes compostos pelos ácidos clorogênico, gálico, protocatequínico e caféico, apresentaram atividade antioxidante similar ao BHA (Rodriguez de Sotillo et al., 1994).

Na tentativa de se elucidar as diferenças de potencial antioxidante existentes entre os ácidos fenólicos, foi realizada uma comparação quantitativa do comportamento cinético da inibição da oxidação de alguns ácidos benzóicos (ácidos p-hidroxibenzóico, vanílico, siríngico, e 3,4-dihidroxibenzóico) e cinâmicos (ácidos p-cumárico, ferúlico, sinápico e caféico) (Marinova \& Yanishlieva, 1992). Concluiu-se que, no caso dos ácidos benzóicos, a hidroxila presente na molécula do ácido $p$-hidroxibenzóico não confere a este nenhuma propriedade antioxidante. Já a metoxila presente com a hidroxila no ácido vanílico confere a ele uma pequena atividade antioxidante. No caso do ácido siríngico, o qual possui dois grupamentos de metoxila, a ação é 
ainda maior. Com referência aos ácidos cinâmicos, a presença de uma metoxila adjacente à hidroxila, como ocorre no ácido ferúlico, aumenta o período de indução da oxidação duas vezes em relação ao controle. Este decurso de tempo é ainda maior com a presença de duas metoxilas, como ocorre no ácido sinápico. Entretanto, o maior potencial antioxidante foi encontrado quando há duas hidroxilas nas posições 3 e 4, estrutura apresentada pelos ácidos caféico e 3,4-dihidroxibenzóico. Portanto, a atividade antioxidante dos compostos estudados por estes autores possuem a seguinte ordem: ácido caféico > 3,4-dihidroxibenzóico > sinápico > siríngico $>$ ferúlico $>p$-cumárico $>$ vanílico.

Em estudos cinéticos mais recentes, utilizando-se triglicerídios e metil ésteres de óleo de girassol, foi verificado que os ácidos fenólicos participam mais efetivamente na fase de iniciação da oxidação e os ácidos ferúlico, caféico e sinápico atuam também nas reações de propagação. Isso leva ao melhor esclarecimento da ação destes antioxidantes no processo oxidativo (Yanishlieva \& Marinova, 1995).

\section{Ácidos fenólicos como antioxidantes biológicos}

Algumas citações foram encontradas com relação à ação antioxidante dos ácidos fenólicos em sistema biológico, e na maioria das vezes foram realizados estudos in vitro com estas substâncias.

Primeiramente, foi verificado o efeito dos ácidos cafeoquínico, caféico e clorogênico na peroxidação in vitro de microssomas (Kimura et al., 1984). Os resultados mostraram eficácia de todas as formas isoméricas do ácido dicafeoquínico na inibição da peroxidação induzida de microssomas, inclusive com ação maior que os ácidos caféico e clorogênico, os quais apresentaram também elevado potencial antioxidante.
Também foram investigados os potenciais antioxidantes dos ácidos caféico e ferúlico in vitro, utilizando microssomas de fígado de ratos (Pulla Reddy \& Lokesh, 1992). Os autores observaram uma inibição significativa da oxidação pelo ácido caféico (86\%), mas quase nenhum poder antioxidante do ácido ferúlico (19\%), pois a atividade deste não foi significativa em relação ao controle.

Em pesquisas realizadas com vários ácidos fenólicos, o clorogênico e o caféico demonstraram uma elevada ação inibitória sobre a peroxidação de células como eritrócitos e monócitos, enquanto os ácidos cinâmico, p-cumárico, ferúlico, protocatequínico e vanílico não apresentaram atividade quando induzidos pelo $\mathrm{H}_{2} \mathrm{O}_{2}$ (Ohnishi et al., 1994; Nardini et al., 1998).

Considerando-se que a modificação oxidativa das lipoproteínas de baixa densidade (LDL) é um iniciador importante da aterogênese (Steinberg et al., 1989; Steinbrecher et al., 1990), vários autores utilizaram o modelo de oxidação de LDL in vitro para verificar o potencial antioxidante de ácidos fenólicos. Alguns deles, como o caféico e o clorogênico, apresentaram um elevado poder antioxidante neste sistema, sendo comparado ao poder encontrado para a vitamina E, C e $\beta$-caroteno (Laranjinha et al., 1994; Vinson et al., 1995; Laranjinha et al., 1996; Vinson \& Dabbagh, 1998).

A maioria dos modelos experimentais empregados na análise do potencial antioxidante de ácidos fenólicos sobre a oxidação de LDL in vitro utilizam o $\mathrm{Cu}^{2+}$ para promovê-la. Neste sistema foi observado um elevado poder antioxidante de ácidos fenólicos como o caféico e o sinápico (Nardini et al., 1995; Carbonneau et al., 1997; Visioli \& Galli, 1998). Contudo, foi descrito que a habilidade do ácido ferúlico neste caso está diretamente ligada ao agente utilizado, pois resultados encontrados em pesquisas demonstraram a ação deste ácido como pró-oxidante quando a oxidação de LDL é induzida pelo cobre, sugerindo um mecanismo no qual há formação de um radical fenoxil ferúlico no 
processo de redução do cobre. Já na oxidação induzida por metamioglobina o ácido ferúlico apresentou um bom efeito antioxidante (Bourne \& Rice-Evans, 1997; Carbonneau et al., 1997).

Avaliou-se também o potencial antioxidante dos ácidos caféico e $p$-cumárico e do ascorbato na oxidação de LDL promovida por ferrilmioglobina (Vieira et al., 1998). Foram observadas atividades antioxidantes por parte dos dois ácidos fenólicos, inclusive maiores que a apresentada pelo ascorbato. Além disso, estas substâncias demonstraram um efeito sinérgico, considerado pelos autores um fato de grande relevância fisiológica.

Embora tenham sido realizados diversos estudos comprobatórios da atividade antioxidante dos ácidos fenólicos in vitro, pouco se sabe a respeito do comportamento destas substâncias in vivo. Há poucas evidências que levam a crer em ação destas substâncias no sistema biológico de forma similar à vitamina $C$ no processo de regeneração do $\alpha$-tocoferol (Nardini et al., 1997).

\section{O NCLUSÃO}

Apesar do conhecimento existente sobre o potencial antioxidante apresentado pelos ácidos fenólicos dos alimentos, é de extrema importância o estudo da ação destas substâncias in vivo, pois não foram encontrados dados a respeito de sua absorção, biodisponibilidade em condições fisiológicas e concentração plasmática ideal para sua atividade de proteção contra os radicais livres e doenças associadas, ainda que tenha sido verificado um elevado potencial antioxidante destes compostos in vitro.

\section{REFERÊ NCIAS BIBLIOGRÁFICAS}

ALLEN, J.C., HAMILTON, R.J. Rancidity in foods. London: Applied Science, 1983. 199p.
ARUOMA, O.I. Free radicals and food. Chemistry in Britain, London, v.29, n.3, p.210-214, 1993.

AZIZ, N.H., FARAG, S.E., MOUSA, L.A., ABO-ZAID, M.A. Comparative antibacterial and antifungal effects of some phenolic compounds. Microbios, Cambridge, v.93, n.374, p.43-54, 1998.

BOURNE, L.C., RICE-EVANS, C.A. The effect of the phenolic antioxidant ferulic acid on the oxidation of low density lipoprotein depends on the pro-oxidant used. Free Radical Research, Chur, v.27, n.3, p.337-344, 1997.

BRAVO, L. Polyphenols: chemistry, dietary sources, metabolism and nutrition significance. Nutrition Reviews, New York, v.56, n.11, p.317-333, 1998.

CARBONNEAU, M.A., LÉGER, C.L., MONNIER, L., BONNET, C., MICHEL, F., FOURET, G., DEDIEU, F., DESCOMPS, B. Supplementation with wine phenolic compounds increases the antioxidant capacity of plasma and vitamin E of low-density lipoprotein without changing the lipoprotein $\mathrm{Cu}^{2+}$ oxidizability: possible explanation by phenolic location. European Journal of Clinical Nutrition, London, v.51, n.10, p.682-690, 1997.

CINTRA, R.M.G., MANCINI FILHO, J. Antioxidant activity of spices in different systems. In: BIENNIEAL MEETING INTERNATIONAL SOCIETY FOR FREE RADICAL RESEARCH, 8., 1996, Barcelona. Abstract Book. Barcelona : ISFRR, 1996. p.90.

COSGROVE, J.P., CHURCH, D.F., PRYOR, W.A. The kinetics of autoxidation of polyunsaturated fatty acids. Lipids, Champaign, v.22, n.5, p.299-304, 1987.

CROFT, K.D. The chemistry and biological effects of flavonoids and phenolic acids. Annals of the New York Academy of Science, New York, v.854, p.435-442, 1998.

DONNELLY, J.K., ROBINSON, D.S. Invited review. Free radical in foods. Free Radical Research, Yverdon, v.22, n.2, p.147-176, 1995.

DURÁN, R.M., PADILLA, B. Actividad antioxidante de los compuestos fenólicos. Grasas y Aceites, Sevilla, v.44, n.2, p.101-106, 1993.

DZIEDZIC, S.Z., HUDSON, B.J.F. Phenolic acids and related compounds as antioxidants for edible oils. Food Chemistry, Oxford, v.14, p.45-51, 1984. 
ERENEL, G., ERBAS, D., ARICIOGLU, A. Free radicals and antioxidant systems. Materia Medica Polona, Warsaw, v.1, n.85, p.37-43, 1993.

FERGUSON, L.R., HARRIS, P.J. Protection against cancer by wheat bran: role of dietary fibre and phytochemicals. European Journal of Cancer Prevention, Oxford, v.8, n.1, p.17-25, 1999.

FERNANDEZ, M.A., SAENZ, M.T., GARCIA, M.D. Antiinflamatory activity in rats and mice of phenolic acids isolated from Scrophularia frutescens. Journal of Pharmacy and Pharmacology, London, v.50, n.10, p.1183-1186, 1998.

FRANKEL, E.N. Lipid oxidation. Progress in Lipid Research, Oxford, v.19, n.1-2, p.1-22, 1980.

GAMACHE, P., RYAN, E., ACWORTH, I.N. Analysis of phenolic and flavonoid compounds in juice beverages using high-performance liquid chromatography with coulometric array detection. Journal of Chromatography, Amsterdam, v.635, n.1, p.143-150, 1993.

HALLIWELL, B., MURCIA, M.A., CHIRICO, S., ARUOMA, O.I. Free radicals and antioxidants in food and in vivo: what they do and how they work. Critical Reviews in Food Science and Nutrition, Cleveland, v.35, n.1/2, p.7-20, 1995.

HO, C.T. Phenolic compounds in food-an overview. In: HO, C.T., LEE, C.Y., HUANG, M.T. Phenolic compounds in food and their effects on health. Washington: American Chemical Society, 1992. p.2-7. (ACS Symposium Series, n.506).

HO, C.T., FERRARO, T., CHEN, Q., ROSEN, R.T., HUANG, T.M. Phytochemicals in teas and rosemary and their cancer-preventive properties. In: HO, C.T., OSAWA, T., HUANG, T.M., ROSEN, R.T. Food phytochemicals for cancer prevention. Washington : American Chemical Society, 1994. p.2-19. (ACS Symposium Series, n.547).

HOLLMAN, P.C., KATAN, M.B. Bioavailability and health effects of dietary flavonoids in man. Archives of Toxicology Supplement, Berlin, v.20, p.237-248, 1998.

HSIEH, R.J., KINSELLA, J.E. Oxidation of polyunsaturated fatty acids: mechanisms, products, and inhibition with emphasis on fish. Advances in
Food and Nutrition Research, San Diego, v.33, p.233-341, 1989.

HUANG, M.T., FERRARO,T. Cancer chemoprevention by phytochemicals in fruits and vegetables: an overview. In: HO, C.T., OSAWA, T., HUANG, T.M., ROSEN, R.T. Food phytochemicals for cancer prevention. Washington : American Chemical Society, 1994. p.2-16. (ACS Symposium Series, n.546).

IVANOVA, A., MILKOVA, T., GALABOV, A.S., NIKOLAEVA, L., VOYNOVA, E. Transformation of cholanic acid derivatives into pharmacologically active esters of phenolic acids by heterogeneous Wittig reaction. Zeitschrift fuer Naturforschung, Tuebingen, v.52, n.7-8, p.516-521, 1997.

KERRY, N.L., ABBEY, M. Red wine and fractionated phenolic compounds prepared from red wine inhibit low density lipoprotein oxidation in vitro. Atherosclerosis, Limerick, v.135, n.1, p.93-102, 1997.

KIMURA, Y., OKUDA, H., OKUDA, T., HATANO, T., AGATA, I., ARICHI, S. Studies on the activities of tannins and related compounds. $V$. Inhibitory effects on lipid peroxidation in mitochondria and microsomes of liver. Planta Medica, Stuttgart, v.50, n.6, p.473-477, 1984.

KING, A., YOUNG, G. Characteristics and occurrence of phenolic phytochemicals. Journal of the American Dietetic Association, Chicago, v.99, n.2, p.213-218, 1999.

KUBOW, S. Lipid oxidation products in food and atherogenesis. Nutrition Reviews, New York, v.51, n.2, p.33-40, 1993.

LARANJINHA, J.A.N., ALMEIDA, L.M., MADEIRA, V.M.C. Reactivity of dietary phenolic acids with peroxyl radicals: antioxidant activity upon LDL oxidation. Biochemical Pharmacology, London, v.48, n.3, p.487-492, 1994.

LARANJINHA, J.A.N., VIEIRA, O., ALMEIDA, L.M., MADEIRA, V.M.C. Inhibition of metmyoglobin/ $\mathrm{H}_{2} \mathrm{O}_{2}$ dependent LDL lipid peroxidation by naturally occuring phenolic acids. Biochemical Pharmacology, London, v.51, n.4, p.395-402, 1996. 
MACHLIN, L.J., BENDICH, A. Free radical tissue damage: protective role of antioxidant nutrients. FASEB Journal, Bethesda, v.1, n.6, p.441-445, 1987.

MARINOVA, E.M., YANISHLIEVA, N.V. Inhibited oxidation of lipids II: Comparison of the antioxidative properties of some hydroxy derivatives of benzoic and cinnamic acids. Fett-Wissenschaft Technologie, Leinfeldem-Echterdingen, v.94, n.11, p.428-432, 1992.

NAGEN, T.J., ALBUQUERQUE, T.T.O., MIRANDA, L.C.G. Ácidos fenólicos em cultivares de soja: ação antioxidante. Arquivos de Biologia e Tecnologia, Curitiba, v.35, n.1, p.129-138, 1992.

NAKATANI, N. Natural antioxidants from spices. In: HO, C.T., LEE, C.Y., HUANG, M.T. Phenolic compounds in food and their effects on health. Washington : American Chemical Society, 1992. p.72-86. (ACS Symposium Series, n.507).

NAMIKI, M. Antioxidants/antimutagens in food. Journal of Nutrition, Boca Raton, v.29, n.4, p.273-300, 1990.

NARDINI, M., D'AQUINO, M., TOMASSI, G., GENTILI, V., Di FELICE, M., SCACCINI, C. Inhibition of human low-density lipoprotein oxidation by caffeic acid and other hydroxycinnamic acid derivatives. Free Radical Biology and Medicine, New York, v.19, n.5, p.541-552, 1995.

NARDINI, M., NATELLA, F., GENTILI, V., Di FELICE, M., SCACCINI, C. Effect of caffeic acid dietery supplementation on the antioxidant defense system in rat: an in vivo study. Archives of Biochemistry and Biophysic, San Diego, v.342, n.1, p.157-160, 1997.

NARDINI, M., PISU, P., GENTILI, V., NATELLA, F., Di FELICE, M., PICCOLELLA, E., SCACCINI, C. Effect of caffeic acid on tert-butyl hydroperoxide-induced oxidative stress in U937. Free Radical Biology and Medicine, New York, v.25, n.9, p. 1098-1105, 1998.

NAWAR, W.W. Lipids. In: FENNEMA, O.R. (Ed.). Food Chemistry. 2.ed. New York : Marcel Dekker, 1985. p.139-244.

OHNISHI, M., MORISHITA, H., IWAHASHI, H., TODA, S., SHIRATAKI, Y., KIMURA, M., KIDO, R. Inhibitory effects of chlorogenic acids on linoleic acid peroxidation and hemolysis. Phytochemistry, Oxford, v.36, n.3, p.579-583, 1994.

ONYENEHO, S.N., HETTIARACHCHY, N.S. Antioxidant activity of durum wheat bran. Journal of Agriculture Food Chemistry, Washington, v.40, p.1496-1500, 1992.

ONYENEHO, S.N., HETTIARACHCHY, N.S. Antioxidant activity, fatty acids and phenolic acids compositions of potato peels. Journal Science Food Agriculture, London, v.62, p.345-350, 1993.

PAPADOPOULOS, G., BOSKOU, D. Antioxidant effect of natural phenols on olive oil. Journal American Oil Chemists' Society, Champaign, v.68, n.9, p.669$671,1991$.

PELEG, H., BODINE, K.K., NOBLE, A.C. The influence of acid on adstringency of alum and phenolic compounds. Chemical Senses, Oxford, v.23, n.3, p.371-378, 1998.

PRATT, D.E. Natural antioxidants from plant material. In: HO, C.T., LEE, C.Y., HUANG, M.T. Phenolic compounds in food and their effects on health. Washington : American Chemical Society, 1992. p.54-71. (ACS Symposium Series, n.507).

PRATT, D.E., BIRAC, P.M. Source of antioxidant activity of soybean and soy products. Journal Food Science, Chicago, v.44, p.1720-1722, 1979.

PULLA REDDY, A.C., LOKESH, B.R. Studies on spice principles as antioxidants in the inhibition of lipid peroxidation of rat liver microsomes. Molecular and Cellular Biochemistry, Norwell, v.111, n.1/2, p.117-124, 1992.

RIBÉREAU-GAYON, P. Les Composés Phénoliques des Végétaux. Paris: Dunod, 1968. 254p.

RICE-EVANS, C., BURDON, R. Free radical-lipid interactions and their pathological consequences. Progress in Lipid Research, Oxford, v.32, n.1, p.71-110, 1993.

RODRIGUEZ DE SOTILLO, D., HADLEY, M., HOLM, E.T. Potato peel waste: stability and antioxidant activity of a freeze-dried extract. Journal Food Science, Chicago, v.59, n.5, p.1031-1033, 1994.

SHAHIDI, F., JANITHA, P.K., WANASUNDARA, P.D. Phenolic antioxidants. CRC Critical Reviews in 
Food Science and Nutrition, Boca Raton, v.32, n.1, p.67-103, 1992.

SIMIC, M.G., JAVANOVIC, S.V. Inactivation of oxygen radicals by dietary phenolic compounds in anticarcinogenesis. In: HO, C.T., OSAWA, T., HUANG, T.M., ROSEN, R.T. (Ed.). Food phytochemicals for cancer prevention. Washington: American Chemical Society, 1994. p.20-33. (ACS Symposium Series, n.546).

STEINBERG, D., PARTHASARATHY, S., CAREW, T.E., KHOO, J.C., WITZTUM, J.L. Beyond cholesterol: modification of low-density lipoprotein that increase its atherogenicity. New England Journal of Medicine, Boston, v.320, n.14, p.915-924, 1989.

STEINBRECHER, U.P., ZHANG, H., LOUGHEED, M. Role of oxidatively modified LDL in atherosclerosis. Free Radical Biology and Medicine, New York, v.9, n.2, p.155-168, 1990.

VIEIRA, O., LARANJINHA, J., MADEIRA, V., ALMEIDA, L. Cholesteryl ester hydroperoxide formation in myoglobin-catalyzed low density lipoprotein oxidation: concerted antioxidant activity of caffeic and p-coumaric acids with ascorbate. Biochemical Pharmacology, London, v.55, n.3, p.333-340, 1998.

VINSON, J.A., DABBAGH, Y.A., SERRY, M.M., JANG, J. Plant flavonoids, especially tea flavonols, are powerful antioxidants using an in vitro oxidation model for heart disease. Journal Agriculture and Food Chemistry, Washington DC, v.43, p.2800-2802, 1995.
VINSON, J.A., DABBAGH, Y.A. Tea phenols: antioxidant effectiveness of teas, tea components, tea fractions and their binding with lipoproteins. Nutrition Research, Elmsford, v.18, n.6, p.1067-1075, 1998.

VISIOLI, F., GALLI, C. The effect of minor constituents of olive oil on cardiovascular disease: new findings. Nutrition Reviews, New York, v.56, n.5, p.142-147, 1998.

Von GADOW, A., JOUBERT, E., HANSMANN, C.F. Comparison of the antioxidant activity of aspalathin with that of other plant phenols of rooibos tea (Aspalathus linearis), $\alpha$-tocopherol, BHT and BHA. Journal Agriculture and Food Chemistry, Washington DC, v.45, p.632-638, 1997.

WILLIAMSON, G., FAULKNER, K., PLUMB, G.W. Glucosinolates and phenolics as antioxidants from plant foods. European Journal of Cancer Prevention, Oxford, v.7, n.1, p.17-21, 1998.

WÜRTZEN, G. Shortcomings of current strategy for toxicity testing of food chemicals: antioxidants. Food Chemistry and Toxicology, Oxford, v.28, n.11, p.743-745, 1990.

YANISHLIEVA, N.V., MARINOVA, E.M. Effects of antioxidants on the stability of triacylglycerols and methyl esters of fatty acids of sunflower oil. Food Chemistry, Oxford, v.54, n.4, p.377-382, 1995.

Recebido para publicação em 30 de setembro de 1999 e aceito em 22 de março de 2001. 